\title{
Correction to: risk factors for cutaneous leishmaniasis in the rainforest of Bolivia: a cross-sectional study
}

Daniel Eid ${ }^{1,2^{*}}$, Miguel Guzman-Rivero ${ }^{1}$, Ernesto Rojas', Isabel Goicolea², Anna-Karin Hurtig², Daniel Illanes ${ }^{1}$ and Miguel San Sebastian ${ }^{2^{*}}$

\section{Correction}

In the original publication of this article [1], the authors noted that some data were incorrect in Table 4. The corrected data are given in this erratum:

Published online: 21 June 2018

\section{Reference}

1. Eid D, et al. Risk factors for cutaneous leishmaniasis in the rainforest of

Bolivia: a cross-sectional study. Trop Med Health. 2018;46:9. https://doi.org/

10.1186/s41182-018-0089-6.

* Correspondence: libremd@gmail.com

${ }^{1}$ Institute of Biomedical Research, Faculty of Medicine, San Simon University,

Aniceto Arce Avenue, 371 Cochabamba, Bolivia

${ }^{2}$ Department of Public Health and Clinical Medicine, Epidemiology and

Global Health, Umea University, Umea, Sweden 
Table 4 Univariate and multivariate analysis of sociodemographic, housing conditions and protective activities associated with $\mathrm{CL}$

\begin{tabular}{|c|c|c|c|}
\hline \multicolumn{2}{|l|}{ Factor } & \multirow{2}{*}{$\begin{array}{l}\text { Unadjusted OR with } 95 \% \mathrm{Cl} \\
1.0\end{array}$} & \multirow{2}{*}{$\frac{\text { Adjusted OR with 95\%C }}{1.0}$} \\
\hline Sex & Female & & \\
\hline & Male & $2.2(1.3-3.7)$ & $3.2(1.6-6.6)$ \\
\hline Age in years & Mean(SD) & $1.0(1.0-1.0)$ & \\
\hline \multirow[t]{2}{*}{ Residence } & Chipiriri community & 1.0 & 1.0 \\
\hline & Ichoa community & $1.9(1.1-3.3)$ & $1.9(0.6-5.7)$ \\
\hline \multirow[t]{2}{*}{ Years of residence } & More than 10 & 1.0 & \\
\hline & Below 10 & $1.3(0.8-2.2)$ & \\
\hline \multirow[t]{2}{*}{ Migrant from high lands } & No & 1.0 & \\
\hline & Yes & $1.1(0.7-1.8)$ & \\
\hline \multirow[t]{2}{*}{ Mother tongue } & Spanish & 1.0 & \\
\hline & Quechua or Aymara & $1.0(0.5-2.1)$ & \\
\hline \multirow[t]{2}{*}{ Farmer } & No & 1.0 & 1.0 \\
\hline & Yes & $1.9(1.2-3.1)$ & $1.7(0.8-3.4)$ \\
\hline \multirow[t]{2}{*}{ Reading } & No & 1.0 & \\
\hline & Yes & $1.6(0.8-3.2)$ & \\
\hline Education in years & Mean(SD) & $1.0(0.9-1.1)$ & \\
\hline \multirow[t]{2}{*}{ Wall material } & Brick or concrete & 1.0 & 1.0 \\
\hline & Wood and others & $1.9(1.1-3.3)$ & $1.0(0.4-2.7)$ \\
\hline \multirow[t]{2}{*}{ Drainage system } & Septic tank & 1.0 & 1.0 \\
\hline & Cesspool and others & $2.3(1.2-4.5)$ & $2.0(0.6-6.8)$ \\
\hline \multirow[t]{2}{*}{ Cooking fuel } & Wood & 1.0 & \\
\hline & Gas & $1.5(0.9-2.8)$ & \\
\hline \multirow[t]{2}{*}{ Number of rooms } & 3 or more & 1.0 & \\
\hline & Fewer than 3 & $1.8(1.1-2.9)$ & \\
\hline \multicolumn{2}{|c|}{ Number of persons per room Mean(SD) } & $1.21(1.04-1.40)$ & $1.2(1.0-1.4)$ \\
\hline \multirow[t]{2}{*}{ Ownership of dogs } & Yes & 1.0 & \\
\hline & No & $1.1(0.7-2.0)$ & \\
\hline \multirow[t]{2}{*}{ Ownership of hens } & No & 1.0 & 1.0 \\
\hline & Yes & $2.5(1.1-5.9)$ & $2.4(0.9-6.1)$ \\
\hline \multirow[t]{2}{*}{ Use of bed net } & Always & 1.0 & \\
\hline & Sometimes & $0.7(0.3-1.6)$ & \\
\hline \multirow[t]{3}{*}{ Use of repellent } & Always & 1.0 & \\
\hline & Sometimes & $5.7(0.7-47.6)$ & \\
\hline & Never & $4.9(0.6-42.0)$ & \\
\hline \multirow[t]{3}{*}{ House fumigations } & Very often or always & 1.0 & \\
\hline & Some times & $1.4(0.7-2.8)$ & \\
\hline & Never & $1.2(0.7-2.2)$ & \\
\hline \multirow[t]{2}{*}{ Sleeping with windows open } & Always & 1.0 & \\
\hline & Sometimes & $1.2(0.7-2.1)$ & \\
\hline
\end{tabular}

